\title{
Doped-Fiber Amplifier Dynamics: A System Perspective
}

\author{
Alberto Bononi, Member, IEEE, and Leslie A. Rusch, Member, IEEE
}

\begin{abstract}
Sun et al. [1] succeeded in reducing the set of coupled first-order nonlinear partial differential equations determining the wavelength-dependent, time-varying amplifier gain into a single ordinary differential equation (ODE). In this paper, we further simplify the ODE bringing into greater evidence the physical meaning of the amplification process, and greatly enhancing the utility of the ODE as an analysis and design tool. We find that the gain dynamics of a doped-fiber amplifier are completely specified by its total number of excited ions $r$, whose time behavior is described by a simple first-order differential equation. We exploit this new understanding of amplifier gain dynamics: 1) to develop an equivalent circuit model for amplifier gain dynamics, 2) to identify that channel addition causes much faster transients than channel dropping in wavelength division multiplexing networks, and 3) to demonstrate that gain excursions can be significant in multichannel packet switching applications, which unlike timemultiplexed signals are characterized by bursts and lulls in communications. We are also able to revisit the most significant previously published results on both steady-state and dynamic analysis of doped-fiber amplifiers with a much more concise and more intuitive derivation.
\end{abstract}

Index Terms-Doped-amplifier gain dynamics, EDFA, packet switching.

\section{INTRODUCTION}

G AIN dynamics of erbium-doped fiber amplifiers (EDFA's) are already of considerable interest in wavelength division multiplexed (WDM) networks, where network reconfigurations or network faults can lead to the adding or dropping of wavelength channels [1]-[4]. As the need for bandwidth grows, optical communications will become more common in network architectures. Transparent wavelength routed optical networks (WRON) will form large meshes of interconnected fiber links and cross connects. Doped-fiber amplifiers will be key components of such networks, and a study of their gain dynamics in a networking scenario is essential for WRON design.

Given the interest in EDFA gain dynamics, much research has been devoted to the solution of the set of coupled firstorder nonlinear partial differential equations determining the wavelength-dependent, time-varying amplifier gain. The complexity of the numerical solution of these equations has motivated efforts to reduce them or to study steady state and

Manuscript received August 1997. This work was supported by the European Community under INCO-DC project 950959 "DAWRON" and by an NSERC Industrial Chair sponsored by Québec-Téléphone.

A. Bononi is with the Università di Parma, Dipartimento di Ingegneria dell'Informazione, Parma I-43100 Italy.

L. A. Rusch is with the Université Laval Département de Génie Électrique et de Génie Informatique, Ste-Foy, P.Q. G1K 7P4 Canada.

Publisher Item Identifier S 0733-8724(98)03330-1. asymptotic solutions. For instance, Saleh et al. [5] eliminated the time dependence of the gain to arrive at a single transcendental equation for the steady state gain. Habbab et al. [6] arrived at a linear approximation for the small signal gain in single-channel amplifiers. More recently, Sun et al. [1] at Bell Laboratories have succeeded in reducing the system of coupled differential equations into a single ordinary differential equation (ODE).

In this paper, we further simplify the ODE identified by Sun et al. [1] bringing into greater evidence the physical meaning of the amplification process, and greatly enhancing the utility of the ODE as an analysis and design tool. We show that the gain dynamics of a doped-fiber amplifier are completely specified by its total number of excited ions, which we call the reservoir $r(t)$, whose time behavior is described by a simple first-order ODE. As Saleh noted in his landmark paper on the steady-state gain equation [5], the power of such a reduced equation is its simplicity. Standard control theory can now be applied to the amplifier, using $r$ as the state variable. The paramount importance of the reservoir in the EDFA analysis was independently found by Sun $e t$ al. in a paper submitted at almost the same time as ours [7].

We note that the present analysis is based on the assumptions of the model in [1] and [5], which neglects both excited state absorption and saturation induced by the amplified spontaneous emission (ASE) produced inside the amplifier. There are several methods to include such ASE contributions in the model [8], [9] and future work will address this issue.

By further reducing the ODE describing the gain dynamics we are able to revisit the most significant previously published results on both steady-state and dynamic analysis of dopedfiber amplifiers with a much more concise and more intuitive derivation. Besides confirming previous results, this analysis tool can now be used to attack open problems and newly emerging issues in doped-fiber amplified communications. The first immediate application of our results is the development of an equivalent circuit model for amplifier gain dynamics. This will allow analysis of extensive chains of amplifiers by the readily available circuit simulation software already in place.

The next result relates to the relative speed of transients in added versus dropped WDM channels. We have found that the doped-fiber amplifier dynamics are connected to the depletion and the refill of the reservoir. While the refill process is mainly contributed by the pump, and is a process in which one pump photon can excite at most one ion, the depletion process is mainly caused by the signals, and is an avalanche process connected to stimulated emission: one signal input photon can 
"burn" a very large number of excited ions in the reservoir. Thus, the time scales connected to the depletion process can be extremely fast, while those connected to the refill process are slow and depend on the pump power and the total number of dopant ions. In any case, the amplifier dynamics are essentially independent of the fluorescence time. As a consequence, channel addition causes much faster transients than channel dropping.

Finally we examine the issue of amplifier response to packet communications, which unlike time-multiplexed signals are characterized by bursts and lulls in communications. We show gain excursions can be significant in multichannel packet switching applications, where signals are kept at a high power level to avoid optical signal-to-noise ratio (SNR) degradations when multiple amplifiers must be crossed.

The paper is organized as follows. In Section II, we derive the ODE describing the system. As applications of our system view of the amplifier, we give: an equivalent electric circuit model of the amplifier (Section III); a view of the gain spread as a function of the state $r$ (Section IV); an analysis of the system reachability space (Section V); the steady-state asymptotic values for large pump (Section VI); analytical approximations to the step response of the system, both for a single amplifier (Section VII); and for a chain of amplifiers (Section VIII); the amplifier gain dynamics in a packet switching scenario (Section IX).

\section{THEORY}

We start from the rate and photon equations used in [1], derived assuming a two-level system for the dopant ions, homogeneously broadened gain spectrum, no excited state absorption, no background loss, and no self-saturation by ASE. The rate equation for the fraction of excited ions $N_{2}$, $0 \leq N_{2} \leq 1$, is

$$
\frac{\partial N_{2}(z, t)}{\partial t}=-\frac{N_{2}(z, t)}{\tau}-\frac{1}{\rho A} \sum_{j=0}^{N} u_{j} \frac{\partial Q_{j}(z, t)}{\partial z}
$$

and the equations describing the propagation along $z$ of the photon fluxes $Q_{k}$ [photons/s] of channel $k, k=0, \cdots, N$, are

$$
\frac{\partial Q_{k}(z, t)}{\partial z}=\rho u_{k} \Gamma_{k}\left[\sigma_{k}^{T} N_{2}(z, t)-\sigma_{k}^{a}\right] Q_{k}(z, t)
$$

where $\tau[\mathrm{s}]$ is the fluorescence time, $\rho\left[\mathrm{m}^{-3}\right]$ is the ion density in the doped fiber core of effective area $A\left[\mathrm{~m}^{2}\right] ; \Gamma_{k}, \sigma_{k}^{e}\left[\mathrm{~m}^{2}\right]$, and $\sigma_{k}^{a}\left[\mathrm{~m}^{2}\right]$ are the confinement factor, and the emission and absorption cross sections of channel $k$, respectively, and $\sigma_{k}^{T} \triangleq \sigma_{k}^{e}+\sigma_{k}^{a}$. The length of the amplifier is $L$ [m]. Channels entering at $z=0$ have $u_{k}=1$ while those entering at $z=L$ have $u_{k}=-1$. The pump is placed on channel 0 .

Dividing both sides of (2) by $Q_{k} \neq 0$, multiplying by $d z$ and integrating from $z=0$ to $L$ yields

$$
G_{k}(t)=B_{k} r(t)-A_{k}, \quad k=0, \cdots, N
$$

where

$$
G_{k}(t) \triangleq \int_{0}^{L} \frac{u_{k} \partial Q_{k}}{Q_{k}}=\ln \left[\frac{Q_{k}^{\text {out }}(t)}{Q_{k}^{\mathrm{in}}(t)}\right]
$$

is the logarithmic gain

$$
r(t) \triangleq \rho A \int_{0}^{L} N_{2}(z, t) d z
$$

is the total number of excited ions in the amplifier, which we call the "reservoir." The reservoir is a number between " 0 " and $r_{M} \triangleq \rho A L$, the total number of ions in the doped fiber. The state variable represents the number of available ions ready to be converted into signal photons

$$
A_{k} \triangleq \rho \Gamma_{k} \sigma_{k}^{a} L \text { and } B_{k} \triangleq \Gamma_{k} \sigma_{k}^{T} / A
$$

are nondimensional parameters.

The standard parameters used in [1]-[6] are the absorption coefficients $\alpha_{k} \triangleq \rho \Gamma_{k} \sigma_{k}^{a}=A_{k} / L$ and the intrinsic saturation powers $P_{k}^{I S} \triangleq h \nu A / \Gamma_{k} \sigma_{k}^{T} \tau=h \nu / B_{k} \tau$. We introduce parameters $A_{k}, B_{k}$, which are independent of $\tau$, to stress that the gain depends on $\tau$ only through $r$. Note that the definitions of $Q_{k}^{\text {in }}(t)$ and $Q_{k}^{\text {out }}(t)$ include the directionality of the fluxes, therefore obviating the need for the parameter $u_{k}$ [1].

Multiplying both sides of (1) by $d z$ and integrating from " 0 " to $L$ yields

$$
\frac{\partial r(t)}{\partial t}=-\frac{r(t)}{\tau}-\sum_{j=0}^{N}\left[Q_{j}^{\text {out }}(t)-Q_{j}^{\text {in }}(t)\right]
$$

Using (3) and (4) we arrive at a first-order ODE describing the dynamic time behavior of the system's state, i.e., the reservoir $r(t)$

$$
\dot{r}(t)=-\frac{r(t)}{\tau}+\sum_{j=0}^{N} Q_{j}^{\text {in }}(t)\left[1-e^{B_{j} r(t)-A_{j}}\right]
$$

Once the initial condition $r(0)$ is specified, it is easy to show that the solution of (5) is unique. $r(0)$ can be any number in the allowed range $\left[0, r_{M}\right]$, although the range spanned by a real amplifier can be narrower, as we will see in Section V. If at time $t=0^{-}$, i.e., one instant before the start of the observation period, the amplifier is at equilibrium, then $r(0)$ must satisfy (5) with $\dot{r}\left(0^{-}\right)=0$

$$
r(0)=\tau \sum_{j=0}^{N} Q_{j}^{\mathrm{in}}\left(0^{-}\right)\left[1-e^{B_{j} r(0)-A_{j}}\right]
$$

which is the well-known Saleh steady-state equation [5]. For a starting guess at its numerical solution, the upper bound $\tau \sum_{j=0}^{N} Q_{j}^{i n}\left(0^{-}\right)$can be used.

Note that, for given input fluxes $Q_{j}^{\text {in }}(t)$, the direction of their entering the amplifier has no effect on $r$. Hence co- and counterpropagating pumping are equivalent in this analysis. However we recall that ASE has been neglected in this analysis, and in fact a copropagating pump always gives a larger optical SNR [10]. 
Equation (5) can be expressed equivalently in terms of the normalized reservoir $x(t) \triangleq 1 / L \int_{0}^{L} N_{2}(z, t) d z=r(t) / r_{M}$, also known as the fraction of excited ions [8], [10],

$$
\dot{x}(t)=-\frac{x(t)}{\tau}+\sum_{j=0}^{N} \frac{Q_{j}^{\mathrm{in}}(t)}{\rho A L}\left\{1-e^{\rho L \Gamma_{j}\left[\sigma_{j}^{T} x(t)-\sigma_{j}^{a}\right]}\right\} .
$$

This form may be more useful in the study of an isolated amplifier, as the main amplifier parameters are clearly visible in the expression.

Note from (7) that input fluxes $Q_{j}^{\text {in }}$ are meaningful in relation to the total reservoir capacity $r_{M}$. However, the normalized input photon fluxes $Q_{j}^{\text {in }} / r_{M}$ do not uniquely describe the system behavior, since amplifiers with identical $\sigma^{T}(\lambda), \sigma^{a}(\lambda)$, but different $r_{M}$ will behave differently even if driven by the same normalized photon fluxes. All other parameters being equal, however, one can trade $\rho$ for $L$ by keeping their product constant, i.e., one can have shorter but otherwise identically behaving amplifiers by more strongly doping the core. Another implication of (7) is that if the core area is doubled the input fluxes must be doubled to obtain identical system dynamics.

\section{EQuivalent Electric Circuit Model}

We know by (5) that the derivative of $r$ exists, hence $r$ is a continuous function of $t$, even when the inputs $\left\{Q_{j}^{\mathrm{in}}\right\}$ are discontinuous. Just as the charge on a capacitor cannot change instantaneously, the reservoir is similarly constrained. Note also that (5) has a nice physical meaning: the variation of the reservoir (the "charge" on the amplifier) is given by the total input flux $\sum_{j=0}^{N} Q_{j}^{\text {in }}$, minus the output flux $\sum_{j=0}^{N} Q_{j}^{\text {out }}$, minus the spontaneous decay from the excited level $r / \tau$. Hence by identifying photon fluxes with currents, and the reservoir with the charge on a capacitor, or better yet with the voltage across a capacitor of capacity $C=1$, we get the equivalent electric circuit depicted in Fig. 1(a).

The circuit is composed of $N$ input current sources (the channels) and of a pump channel " 0 ," hidden inside the amplifier. The currents feed the capacitor, whose voltage is the reservoir $r$. The currents out $Q_{j}^{\text {out }}=Q_{j}^{\text {in }} e^{G_{j}(r)}$ are current generators controlled by voltage $r$ and current $Q_{j}^{\text {in. The output }}$ pump current is shunted to ground and does not exit the amplifier. The RC constant of the capacitive circuit is $\tau$. However this is an active circuit, and it is clear that the actual time constants involved in the dynamics are essentially independent of $\tau$.

The circuit model for a subsequent loss $L$ is shown in Fig. 1(b). It is a bank of current-controlled current generators that shunt part of the output photons to ground, effectively wasting them. Using these building blocks for the amplifier and the loss, a complex network of amplifiers can be solved by any electric circuit simulator available on the market. This is perhaps the most immediate practical application of our system view.

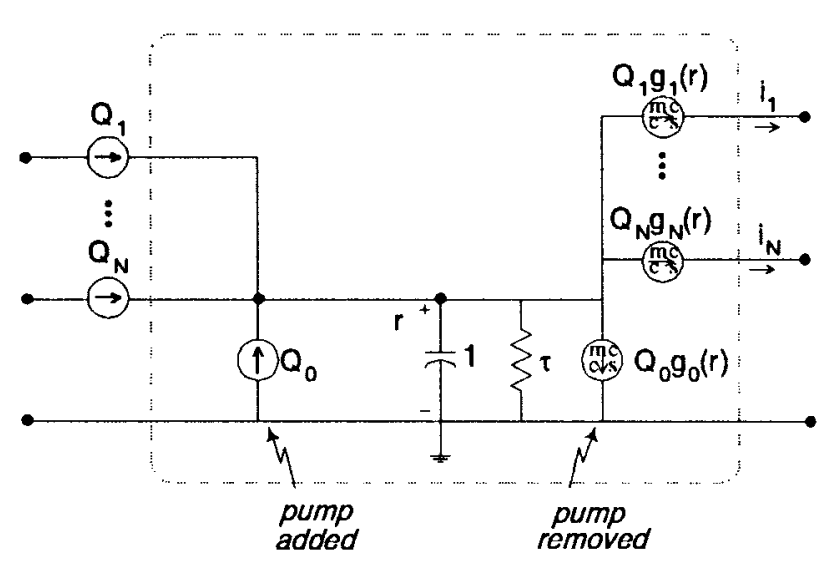

(a)

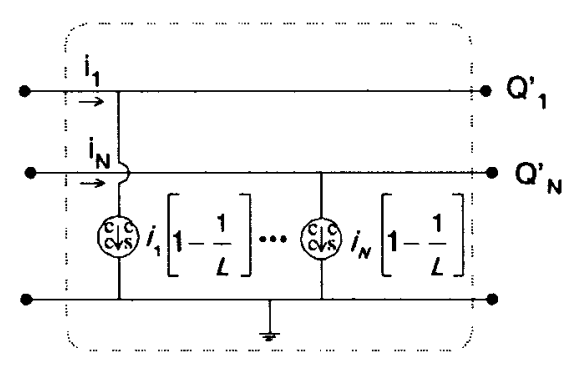

(b)

Fig. 1. (a) Fiber amplifier equivalent circuit and (b) equivalent circuit of an optical loss $L$. Input sources are independent current generators. The controlled generators are cccs $=$ current-controlled current source; mccs $=$ multicontrolled current source.

\section{GAIN SPREAD}

Equation (3) shows that the log-gains of all channels at all times $t$ are linear functions of the (normalized) reservoir $x$

$$
G_{k}(t)=\rho L \Gamma_{k}\left(\sigma_{k}^{T} x(t)-\sigma_{k}^{a}\right)
$$

If we fix a specific choice of cross sections $\sigma^{e}(\lambda), \sigma^{a}(\lambda)$, and plot the quantity $\left\{\sigma^{T}(\lambda) x-\sigma^{a}(\lambda)\right\}$ as a function of $x$, we find that for $x<0.35$, all log-gains are negative, while all $\log$-gains are positive for $x>0.45$. A minimum spread point near $x=0.78$ is clearly visible.

The dB-gain is linearly dependent on the mean fraction of excited ions $x$, i.e., for a specific choice of amplifier parameters, we note again that the gains have minimum spread of about $0.6 \mathrm{~dB}$ for $x$ near 0.78 . As $x$ moves away from such value, the gain spread becomes larger. This view of the linear dependence of the gains on the system state is of paramount importance, as we will see in the following sections.

\section{REACHABILITY}

Once the initial condition $x_{0}$ is given, we have seen that the solution to (7) is unique. What happens if we artificially start the state $x_{0}$ outside the allowed range $[0,1]$ ? What are the minimum and maximum values of $x$ actually reachable by starting the system at equilibrium and by using proper driving signals $Q_{j}^{\mathrm{in}}(t)$ ? Such questions naturally arise when implementing the electric equivalent circuit. 
1) Upper Limit: Suppose we start the system at $x_{0}>1$. Then $\forall j, \sigma_{j}^{T} x_{0}-\sigma_{j}^{a}>\sigma_{j}^{T}-\sigma_{j}^{a}=\sigma_{j}^{e}>0$, and the gain of every channel $j$ is positive, i.e., all channels are gaining photons. Thus, $\forall j, \forall Q_{j}^{\text {in }}$ we have $\left[1-e^{G_{j}(0)}\right]<0$ so that from (7) we have $\dot{x}(0)<0$, i.e., the reservoir must be supplying the required photons and is thus discharging.

The reservoir can only cease discharging when at least one of the gains becomes negative. This happens for the pump first, which is the one providing gain to the channels in normal conditions. Hence, certainly $\dot{x}(t)<0$ until $\sigma_{p}^{T} x-\sigma_{p}^{a} \leq 0$, i.e., until

$$
x \leq x_{U} \triangleq \frac{\sigma_{p}^{a}}{\sigma_{p}^{a}+\sigma_{p}^{e}}<1
$$

which is thus an upper bound on the maximum achievable $x .{ }^{1}$

The actual maximum value of $x$, for a given set of input fluxes $\left\{Q_{j}^{\mathrm{in}}\right\}$, at which the derivative $\dot{x}(t)$ can be zero must be a solution of Saleh's (6). Its maximum value over all possible inputs $\left\{Q_{j}^{\mathrm{in}}\right\}$, for a given finite pump $Q_{p}$, is found by taking $\lim Q_{j}^{\text {in }} \rightarrow 0$ for all $j$, i.e., as the small signal steady-state value $x_{0}^{s s}\left(Q_{p}\right)$ satisfying (6)

$$
x_{0}^{s s}\left(Q_{p}\right)=\tau \frac{Q_{p}}{r_{M}}\left\{1-e^{\rho L \Gamma_{p}\left[\sigma_{p}^{T} x_{0}^{s s}\left(Q_{p}\right)-\sigma_{p}^{a}\right]}\right\} .
$$

The maximum value of $x_{0}^{s s}\left(Q_{p}\right)$ is found letting $Q_{p} \rightarrow \infty$. Since $x$ is bounded, from (9) we must have $1-e^{G_{p}} \rightarrow 0$ as $Q_{p} \rightarrow \infty$. Hence

$$
\lim _{Q_{p} \rightarrow \infty} x_{0}^{s s}\left(Q_{p}\right)=\frac{\sigma_{p}^{a}}{\sigma_{p}^{T}}
$$

i.e., $x_{U}$ is achieved asymptotically for infinite pump.

2) Lower Limit: Suppose we start the system at $x_{0}<0$. Then $\forall j, \sigma_{j}^{T} x_{0}-\sigma_{j}^{a}<-\sigma_{j}^{a}<0$, and the gain of every channel $j$ is negative, i.e., all channels are losing photons. Thus $\forall j, \forall Q_{j}^{\text {in }}$ we have $\left[1-e^{G_{j}(0)}\right]>0$ so that from (7) we have $\dot{x}(0)>0$, and the reservoir is charging. The reservoir will not cease charging until (at least) $x(t)=0$, at which point the derivative could change sign. The lowest achievable value of $x$ is trivially zero, obtained when no beam is present at the input. ${ }^{2}$

In summary, the reachable range of $x$ is $\left[0, x_{U}\right]$. If the amplifier is pumped but the input signals are not present, the (normalized) reservoir $x$ fills up to its rightmost limit. As soon as signal fluxes arrive, the reservoir starts depleting, $x$ starts decreasing, and it will decrease down to its steady state leftmost value if the input signals eventually settle to a limiting continuous-wave $(\mathrm{CW})$ value.

\section{Steady-State Asymptotic Values}

Consider a single fiber amplifier, with $\mathrm{CW}$ input fluxes $Q_{j}^{\text {in }}, j=1, \cdots, N$, and pump flux $Q_{p}$. The steady-state

\footnotetext{
${ }^{1}$ More generally we should write $x \leq \max _{\{j\}}\left\{\sigma_{j}^{a} / \sigma_{j}^{T}\right\}$.

${ }^{2} \mathrm{We}$ are neglecting the thermally induced ion excitation.
}

reservoir value $r^{s s}$ is found from (6) as the solution to

$$
\frac{r^{s s}}{\tau}=Q_{p}\left(1-e^{B_{p} r^{s s}-A_{p}}\right)+\sum_{j=1}^{N} Q_{j}^{\mathrm{in}}\left(1-e^{B_{j} r^{s s}-A_{j}}\right) .
$$

Now we determine the limiting value of $r^{s s}$ when $Q_{p} \rightarrow \infty$, assuming such a limit exists. Take $\lim _{Q_{p} \rightarrow \infty}$ of both sides of (11). A necessary and sufficient condition for the RHS to be finite is $\lim _{Q_{p} \rightarrow \infty} B_{p} r^{s s}-A_{p}=0$ which implies

$$
\lim _{Q_{p} \rightarrow \infty} r^{s s}=\frac{A_{p}}{B_{p}}=\frac{\sigma_{p}^{a}}{\sigma_{p}^{T}} r_{M} .
$$

The log-gain values for infinite pump are therefore

$$
\lim _{Q_{p} \rightarrow \infty} G_{j}=B_{j} \frac{A_{p}}{B_{p}}-A_{j}=\rho L \Gamma_{j}\left(\sigma_{j}^{T} \frac{\sigma_{p}^{a}}{\sigma_{p}^{T}}-\sigma_{j}^{a}\right)
$$

which is a generalization to the multibeam case of the so called infinite-pump small-signal gains calculated by Habbab et al. [6] for a single channel. It is clear that an infinite pump makes any finite beams $\left\{Q_{j}^{\text {in }}\right\}$ look like small signals. For a strong pump, Habbab's linear approximation tends to be more accurate ([6, Figs. 4-6]), and in the infinite pump limit it is exact, since the gains, as seen in (13), do not depend on the signal input power.

\section{GAIN Dynamics: SteP Response}

Suppose we have a single amplifier, described by (5). At time $t=0^{-}$(i.e., an instant before time 0 ) we are given the initial value $r(0)$ and the inputs $\left\{Q_{j}^{\text {in }}\left(0^{-}\right)\right\} .^{3}$ The system need not be at steady state at $t=0^{-}$. At time $t=0^{+}$each input flux undergoes a discontinuity and then remains constant for all $t>0$

$$
Q_{j}^{\text {in }}(t)=Q_{j}^{\text {in }}\left(0^{-}\right)+\Delta Q_{j}^{\text {in }} \quad j=0, \cdots, N .
$$

Then (5) at $t=0^{-}$gives

$$
\dot{r}\left(0^{-}\right)=-\frac{r(0)}{\tau}+\sum_{j=0}^{N} Q_{j}^{\mathrm{in}}\left(0^{-}\right)\left[1-e^{G_{j}(0)}\right]
$$

where $G_{j}(0)=B_{j} r(0)-A_{j}$, and at $t=0^{+}$gives

$$
\dot{r}\left(0^{+}\right)=\dot{r}\left(0^{-}\right)+\sum_{j=0}^{N} \Delta Q_{j}^{\mathrm{in}}\left[1-e^{G_{j}(0)}\right] .
$$

For small values of $t$, we can approximate the actual solution of (5) with a straight line

$$
r(t) \cong r(0)+\dot{r}(0) t .
$$

More generally, we can differentiate (5) $n-1$ times and find the derivatives at time zero $\left(\partial^{i} / \partial t^{i}\right) r\left(0^{+}\right), i=1, \cdots, n$, and approximate $r(t)$ with its truncated Taylor series in $t=0$

$$
r(t) \cong \sum_{i=0}^{n} \frac{\partial^{i}}{\partial t^{i}} r\left(0^{+}\right) \frac{t^{i}}{i !} .
$$

One problem with such polynomial approximations at $t=0$ is that they fail to converge to the actual solution for $t \rightarrow \infty$.

${ }^{3}$ Note that $r\left(0^{+}\right)=r\left(0^{-}\right)$since $\dot{r}(0)$ exists. 
A reasonable compromise between accuracy near $t=0$ and asymptotic convergence is obtained by the exponential approximation

$$
r(t) \cong r^{s s}(\infty)+\left[r(0)-r^{s s}(\infty)\right] e^{-t / \tau_{e}}
$$

where $r^{s s}(\infty)$ is the steady state value of $r$ (approached asymptotically for $t \rightarrow \infty$ ) with the new input fluxes (14), and $\tau_{e}$ is the exponential time constant. To get accuracy near $t=0$ we impose that $\dot{r}_{e}\left(0^{+}\right)=\dot{r}\left(0^{+}\right)$, i.e., the derivative of the approximation matches that of the actual solution at time $t=0^{+}$. We have from (19) $\dot{r}\left(0^{+}\right) \cong-\left[r(0)-r^{s s}(\infty)\right] / \tau_{e}$, so that we find the time constant

$$
\tau_{e}=\frac{r^{s s}(\infty)-r(0)}{\dot{r}\left(0^{+}\right)} \text {. }
$$

The exponential approximation gives a better model to describe the gain dynamics for longer time scales with respect to the polynomial approximations. It gives a good description of the transients in a circuit switching scenario, with channels being added/dropped dynamically. Such an approximation has been suggested in [3], but an explicit expression for the exponential time constant was missing. It has been shown in [3] that the exponential approximation is indeed closer to the experimentally measured value of $r(t)$. Appendix A gives an analytical justification of the exponential approximation, corroborating the results in [4].

As a numerical example, consider a case very similar to the one presented in [1]. The amplifier has two input channels $\lambda_{1}=1552.4 \mathrm{~nm}$ and $\lambda_{2}=1557.9 \mathrm{~nm}$, with initial input powers $P_{1}=-2 \mathrm{dBm}$ and $P_{2}=-2+10 \log _{10}(7) \mathrm{dBm}$, simulating the remaining seven channels of an eight-channel system with $-2 \mathrm{dBm} / \mathrm{channel}$. The amplifier is pumped at 980 $\mathrm{nm}$, with pump power $18.4 \mathrm{dBm}$, and has $L=35 \mathrm{~m}, \tau=10.5$ $\mathrm{ms}$. The absorption coefficients are $[0.257,0.145,0.125] \mathrm{m}^{-1}$ and the intrinsic saturation powers are [0.440, 0.197, 0.214] $\mathrm{mW}$ at $[980,1552.4,1557.9] \mathrm{nm}$, respectively. The system is at equilibrium before $t=0$. At time $t=0$ part of the power on channel 2 is dropped, simulating the drop of a given number of channels.

Fig. 2(a) shows the reservoir dynamics for the exact solution of (5) and for the exponential approximation (19), for power variations in channel 2 simulating the drop of four and seven channels, and the addition of seven channels, respectively. Fig. 2(b) shows the corresponding output power excursion on channel 1 , defined as $10 \log _{10}\left[Q_{1}^{\text {out }}(t) / Q_{1}^{\text {out }}\left(0^{+}\right)\right]$. This figure matches very well with [1, Fig. 1]. The exponential approximation for the reservoir is always below the actual solution, and larger errors are obtained for larger power drops.

As another example of step response, consider in Fig. 3 the turn-on dynamics of the previous amplifier, in which no beam is present before $t=0$ and the pump is turned on at $t=0^{+}$. From (20) we have $\tau_{e}=\left[r^{s s}(\infty)-r(0)\right] / Q_{p}\left[1-e^{G_{p}(0)}\right]=$ $595 \mu$ s. Since we have a strong pump and no signals in at $t<0$, the steady state value is approximated by (12). Observing also that $r(0) \cong 0$ and $e^{G_{p}(0)} \cong 0$ (amplifier OFF), and that $\sigma_{p}^{a} / \sigma_{p}^{T} \cong 1$, we get $\tau_{e} \cong r_{M} / Q_{p}$. This means that the turn-on time is the time it takes the pump, providing $Q_{p}$

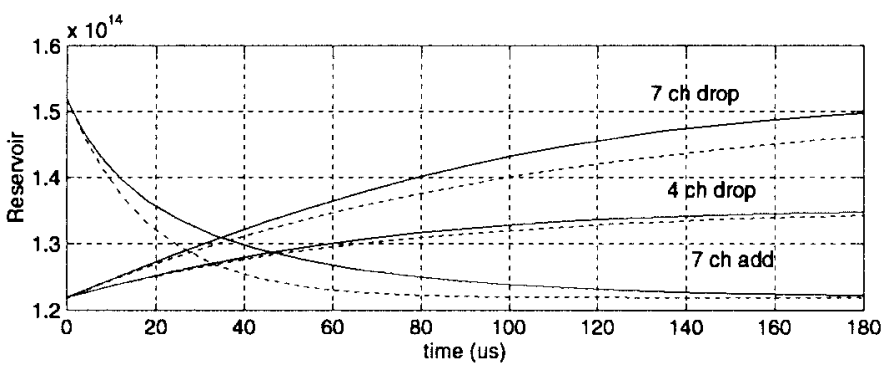

(a)

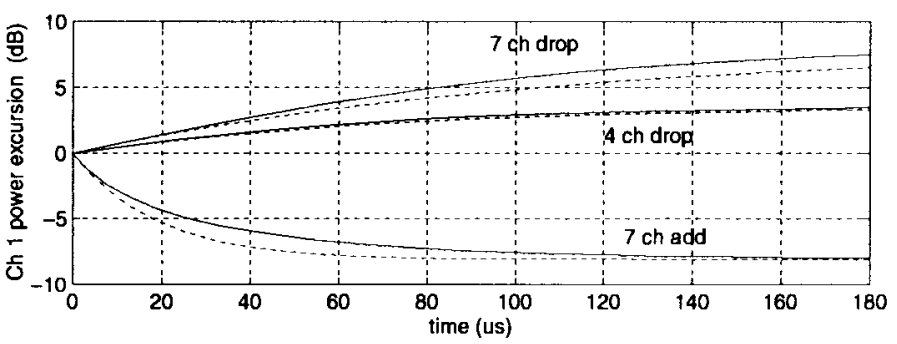

(b)

Fig. 2. Time evolution of (a) reservoir and (b) output power excursion for surviving channel 1 with channel 2 undergoing step variations at $t=0$. Solid: Exact solution of (5). Dashed: Exponential approximation.

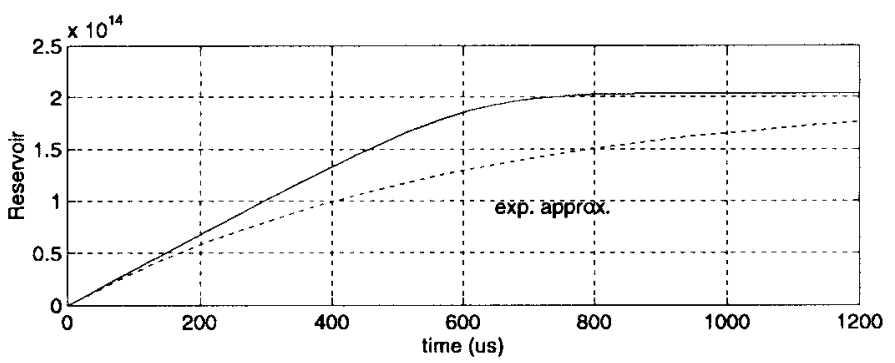

(a)

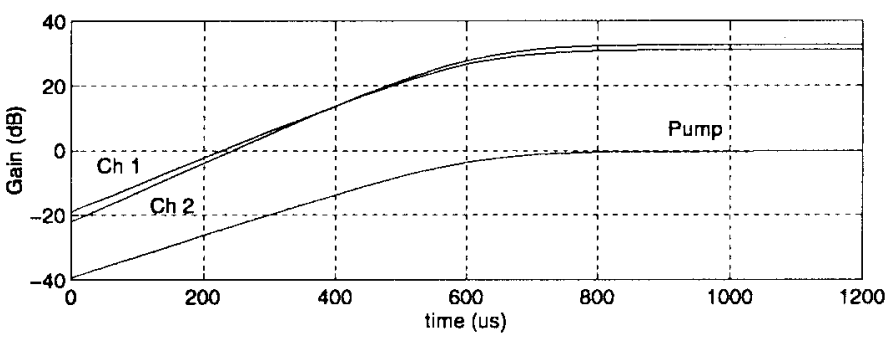

(b)

Fig. 3. Numerical solution of (5) giving the system time evolution, with pump turned on at $t=0$ : (a) reservoir; (b) gains at [980, 1552.4, and 1557.9] nm.

photons/s, to invert the whole ion population in a one-to-one process. This also justifies the observed nearly linear increase in the reservoir. Note that the exponential approximation is accurate for low values and high values of $t$, with poorest performance at the knee of the curve. This is not surprising, as the time constant was chosen to give exact results for $t=0$ and $t \rightarrow \infty$.

This long time constant, as well as the ones relative to channel drops in the previous example, which are of the order of $100 \mu \mathrm{s}$, are connected to the long time required by the pump to refill the reservoir. One pump photon can at most excite one 
ion, and thus it takes a strong pump flux to have a fast refill. On the other hand, the fast dynamics observed in the signal add process are connected to the time it takes the added signal to deplete the reservoir. One signal photon can "burn" many excited ions in the stimulated avalanche process, and thus it is sufficient to have a relatively weaker signal to have much faster system dynamics.

\section{SteP RESPONSE IN AMPLIFIER CHAINS: INITIAL POWER EXCURSION}

In this section we apply our theory to the study of transient gain dynamics in a chain of amplifiers in response to channel dropping/adding in a circuit switching scenario, a case also studied in [2]. In Appendix B we further examine how the phenomenon of gain peaking can be predicted using the simplified ODE in (5).

Consider a chain of $m$ identical fiber amplifiers, identical interamplifier loss $L_{I}$, and $N+1$ beams at the chain input, with CW fluxes $Q_{1}, \cdots, Q_{N}$ for the signals, and $Q_{0} \triangleq Q_{p}$ for the pump. As in Section VIII, at time $t=0^{+}$the fluxes at the input of the chain have a discontinuity, so that for $t>0^{+}$ (14) holds for $j=1, \cdots, N$. Such discontinuity propagates instantly along the amplifier chain. ${ }^{4}$ Equations (15) and (16) describe the derivative of reservoir $r_{i}(t)$ at each amplifier $i=1, \cdots, m$ along the chain.

Let $Q_{i j}^{\text {in }}$ be the flux of channel $j$ at the input of the $i$ th amplifier. By definition, $Q_{1, j}^{\text {in }}=Q_{j}^{\text {in }}$ for all $j$, and $Q_{i 0}^{\text {in }}=Q_{p}$ for all $i$ since the pump is restored at each amplifier. Let $g_{i j} \triangleq e^{B_{j} r_{i}-A_{j}}$ be the linear gain of channel $j$ at the $i$ th amplifier. At the input of the $m$ th amplifier, we can thus write for channel $j$

$Q_{m j}^{\mathrm{in}}=Q_{j}^{\mathrm{in}}\left[\prod_{i=1}^{m-1} \frac{g_{i j}}{L_{I}}\right]=Q^{\mathrm{in}}\left[e^{B_{j} \sum_{i=1}^{m-1} r_{i}-(m-1)\left(A_{j}+\ln L_{I}\right.}\right]$.

Equation (16) for the $m$ th amplifier then becomes

$$
\dot{r}_{m}\left(0^{+}\right)=\dot{r}_{m}\left(0^{-}\right)+\sum_{j=1}^{N} \Delta Q_{j}^{\text {in }}\left[\prod_{i+1}^{m-1} \frac{g_{i j}(0)}{L_{I}}\right]\left(1-g_{m j}(0)\right) \text {. }
$$

If the system is started at equilibrium $\left(\dot{r}_{m}\left(0^{-}\right)=0\right)$, and if the gains of each channel are almost equal along the chain, very close to the interamplifier loss and large $\left.\forall i, g_{i j}(0) \triangleq g_{j}(0) \cong L_{I} \gg 1\right)$, then

$$
\dot{r}_{m}\left(0^{+}\right) \cong-\sum_{j=1}^{N} \Delta Q_{j}^{\text {in }} g_{j}(0)
$$

which shows that the slope $\dot{r}_{m}\left(0^{+}\right)$of every reservoir in an initially "balanced" chain is approximately equal to (23) for every amplifier.

As a numerical example, consider a case very similar to the one presented in [2]. The amplifier chain has 20 identical amplifiers and two input channels at 1552.1 and $1557.7 \mathrm{~nm}$,

\footnotetext{
${ }^{4}$ We are neglecting the propagation delay of light in the fiber chain.
}

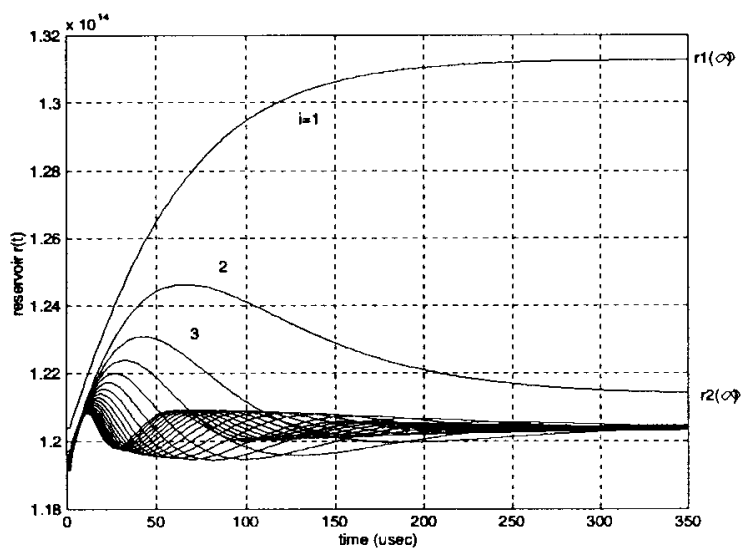

(a)

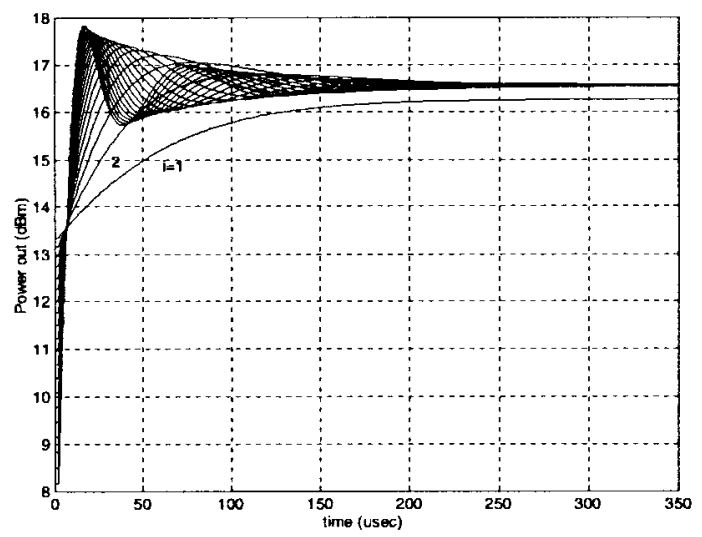

(b)

Fig. 4. Numerical solution of (5) giving the time evolution along a chain of 20 amplifiers with one channel drop at $t=0$ : (a) reservoir and (b) output power of surviving channel. $i=$ amplifier index along the chain.

with initial input power of $3 \mathrm{dBm} / \mathrm{channel}$. The amplifiers are identical to those used in Section VII. The interamplifier loss is $L_{I}=10.32 \mathrm{~dB}$. The system is at equilibrium before $t=0$. At time $t=0$ the $1557.7 \mathrm{~nm}$ channel is dropped completely.

Fig. 4(a) shows the time evolution of the reservoirs $r_{i}(t), i=1, \cdots, 20$ along the chain, obtained by numerically solving (5) for each amplifier. It can be seen that, in this initially well-balanced chain, the initial slopes of all reservoirs are very close in value confirming (23). The first amplifier reservoir has a monotone increase, while all the following amplifiers have damped oscillations in their reservoirs. The new steady-state reservoir values quickly converge beyond the third amplifier $(i>3)$ to the asymptotic value $r_{\infty}^{s s}=\left(A_{1}+\ln L_{I}\right) / B_{1}=1.2039 \times 10^{14}$ determined by channel 1, as derived in (33) of Appendix B.

In the case just examined we have only one signal, and the convergence of the signal gain to the interamplifier loss is a regulating effect known as self-healing [11]. In the case of many signals, Appendix B shows that along an infinite chain only one channel survives, whose gain adjusts to the interamplifier loss, while all the other channels are suppressed.

Fig. 4(b) shows the output powers at channel 1 corresponding to the reservoirs in Fig. 4(a). A strong overshoot is visible as the amplifier index $i$ increases. 


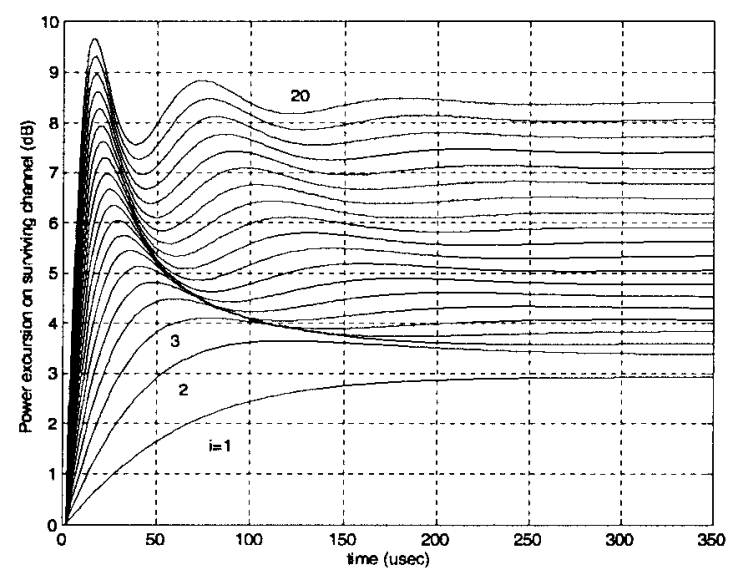

(a)

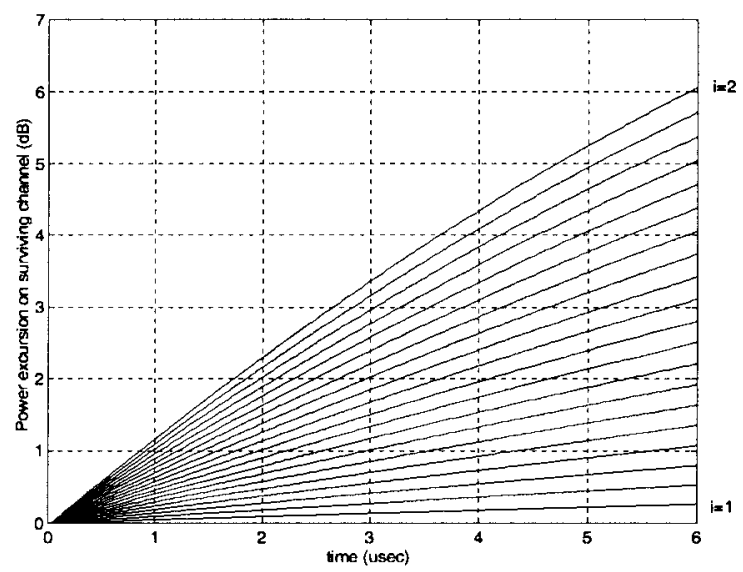

(b)

Fig. 5. Time evolution of the $\mathrm{dB}$ power execution along a chain of 20 amplifiers on the surviving channel. (a) Long time scale and (b) short time scale.

The $\mathrm{dB}$ power excursion at the $m$ th amplifier on channel $s$ is $\Delta P_{m, s}^{\text {out }}(t) \triangleq 10 \log _{10}\left(P_{m, s}^{\text {out }}(t) / P_{m, s}^{\text {out }}\left(0^{+}\right)\right)$[2]. We easily find

$$
\frac{P_{m, s}^{\text {out }}(t)}{P_{m, s}^{\text {out }}\left(0^{+}\right)}=\frac{g_{m, s}(t)}{g_{m, s}(0)} \frac{P_{m-1, s}^{\text {out }}(t)}{P_{m-1, s}^{\text {out }}\left(0^{+}\right)}=\prod_{i=1}^{m} \frac{g_{i s}(t)}{g_{i s}(0)} .
$$

Using the linear approximation $r_{i}(t) \cong r_{i}(0)+\dot{r}_{i}\left(0^{+}\right) t$ for each reservoir and (3), we finally get the $\mathrm{dB}$ power excursion at the $m$ th amplifier as

$$
\Delta P_{m, s}^{\text {out }}(t)=10 \log _{10}(e) B_{s} \sum_{i=1}^{m} \dot{r}_{i}\left(0^{+}\right) t
$$

which shows the excursion grows (initially) linearly in time with slope proportional to the sum of the $t=0^{+}$slopes of the reservoirs along the chain. As noted in [2], if these are all equal, the slope of the excursion grows linearly with the amplifier index down the chain. Fig. 5(a) and (b) shows the power excursion on the surviving channel relative to the example above, respectively, on a long time scale and a short time sclae.

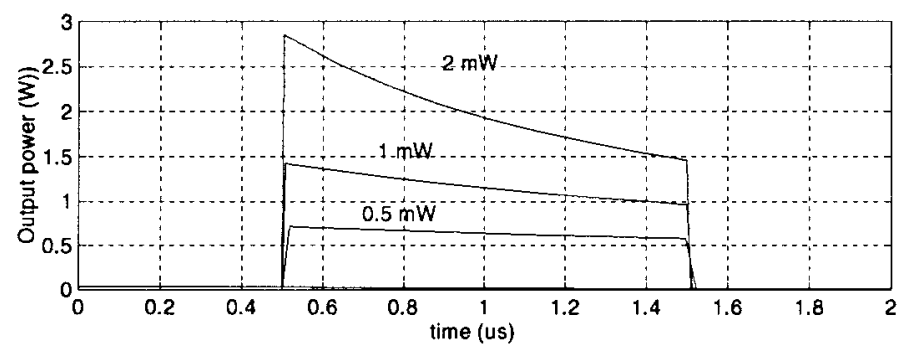

(a)

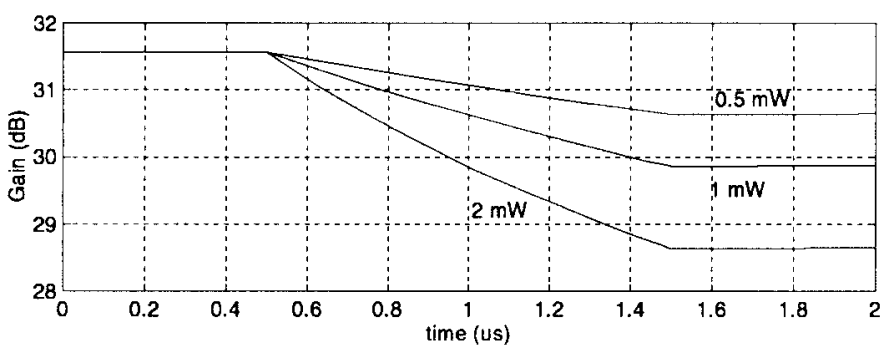

(b)

Fig. 6. Numerical solution of (5) giving the system time evolution with a single pulsed input signal. (a) The graph shows signal and pump outputs powers. (b) The graph shows the signal gain. Input signal powers are 0.5, 1 , and $2 \mathrm{~mW}$.

\section{Dynamics In Packet Switching}

In this section, we will illustrate the amplifier dynamics in a packet switching environment. Bits on the signal channels arrive in bursts, or packets, followed by lulls, i.e., periods of source inactivity.

Assume we start at $t=0$ with the pump only. The first packet reaching the amplifier will find a full reservoir, and thus enjoys the small-signal gain (13) if the pump is strong enough. However, if the input packet power is high and its duration is long, it will quickly start depleting the reservoir by stimulated emission, thus causing a very fast power transient across the packet. The last bits will experience a much lower gain than the initial bits.

As a numerical example, consider a case similar to the one presented in [12]. The amplifier is pumped at $1480 \mathrm{~nm}$, with pump power $36 \mathrm{~mW}$, and has $L=100 \mathrm{~m}, \tau=12 \mathrm{~ms}$. There is one pulsed input channel at $\lambda_{1}=1535 \mathrm{~nm}$, with peak input pulse power varied from 0.5 to $2 \mathrm{~mW}$. The absorption cross sections are $[7.9,1.6] \times 10^{-25} \mathrm{~m}^{2}$ and the emission cross sections are $[0.35,1.7] \times 10^{-25} \mathrm{~m}^{2}$ at $[1480,1535] \mathrm{nm}$, respectively. The system is at equilibrium before $t=0$. At time $t=0.5 \mu \mathrm{s}$ a pulse of duration $1 \mu \mathrm{s}$ arrives on channel 1 .

We can clearly observe in Fig. 6 the power sag across the output packet caused by the fast depletion of the reservoir by the pulse, and the gain decrease across the packet time. A larger signal power gives more power sag. The pump power is also shown, but is barely visible in the Fig. 6; it also exhibits a similar sag in power. Again, the important point is that the gain dynamics can be extremely fast upon strong signal pulse arrivals, due to the stimulated-emission avalanche depletion of the reservoir.

In passing, note that Ko et al. [12] arrived at the same results by solving the system of coupled differential equa- 


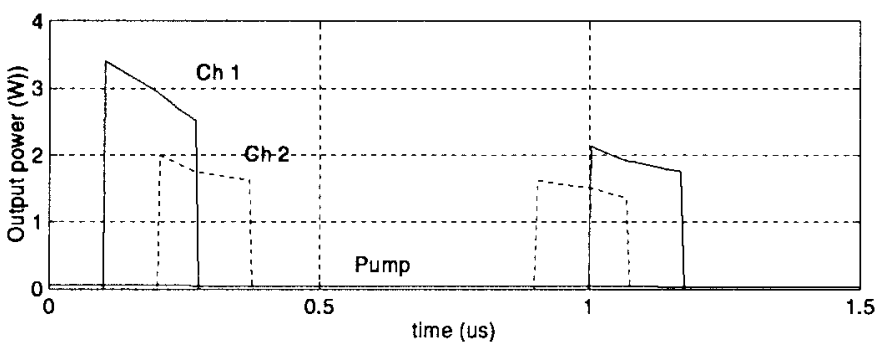

(a)

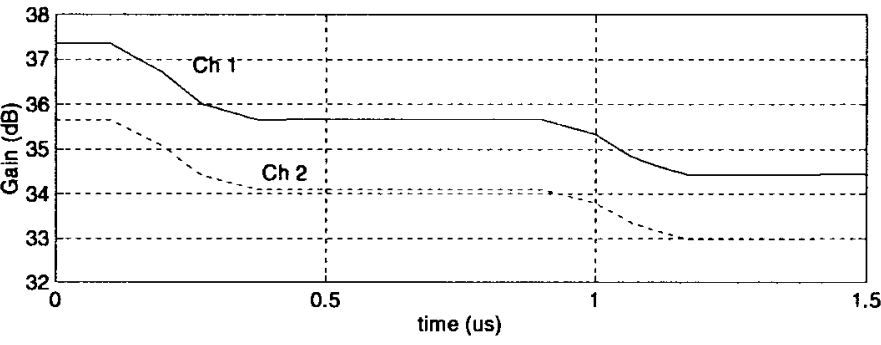

(b)

Fig. 7. Numerical solution of (5) giving the system time evolution with two input pulsed channels of input power $-2 \mathrm{dBm}$ each. Amplifier data are the same as in Fig. 2, but with length $L=40 \mathrm{~m}$. (a) shows pump and signals output powers and (b) shows the gain at the signal wavelengths.

tions describing more accurately the amplifier dynamics. They considered also the effect of spontaneous emission noise, but their results match very well with our figures, indicating that saturation induced by ASE is not an issue here.

Giving the reservoir enough time to refill will effectively restore the initial conditions, so that the next pulse will behave exactly as the one shown in Fig. 6. If however a packet arrives before complete gain recovery (recall that the recovery time is slow, since it is connected to the one-to-one inversion process), it will experience less initial gain, and thus less power sag shows across the output packet power.

As another example, consider the amplifier with two channels used to generate Fig. 2, but with length increased to $L=40 \mathrm{~m}$. As shown in Fig. 7, two pulsed input channels of peak input power $-2 \mathrm{dBm}$ each feed the amplifier, initially at steady state without input signals. Pulses have a duration of $170 \mathrm{~ns}$, corresponding to an asynchronous transfer mode (ATM) cell of 424 bits at $2.5 \mathrm{~Gb} / \mathrm{s}$. The first pulse (packet) arrives on channel 1 and starts depleting the reservoir. When the next pulse on channel 2 arrives and overlaps the first pulse, the reservoir depletes faster, and the gain and power curves become steeper. When the overlapping ends at the end of the first pulse, the slope relaxes, since less reservoir ions get burned per second. When the next pulses arrive, the reservoir has not had time to recover. Actually, the reservoir barely started refilling, as seen by the slight increase of the gain between pulses.

The pump power of $69 \mathrm{~mW}(18.4 \mathrm{dBm})$ is barely visible at the top of Fig. 7. The output power of the first pulses ranges between 2 and $3 \mathrm{~W}$, as should not be surprising. The effect is very similar to Q-switching [13]. Such pulses find the reservoir completely filled, and thus experience the small-signal gain. As subsequent pulses arrive, they will get

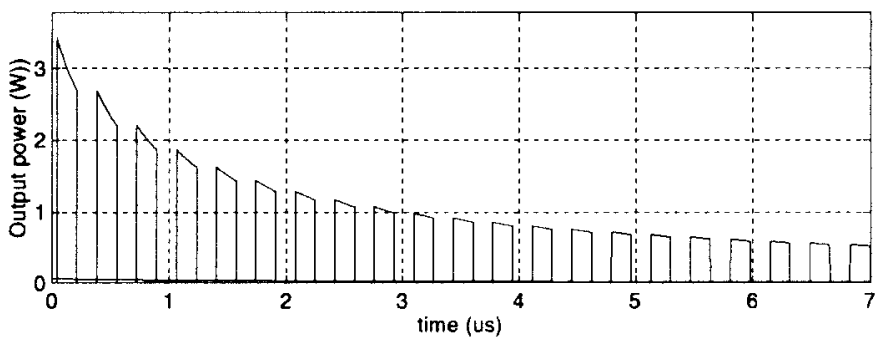

(a)

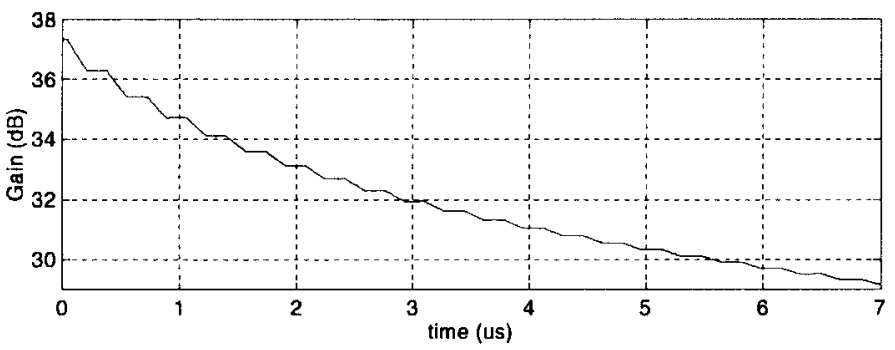

(b)

Fig. 8. Numerical solution of (5) giving the system time evolution with one input train of pulses of peak power $-2 \mathrm{dBm}$, pulse duration $T_{p}=170 \mathrm{~ns}$, one pulse every two slots. Amplifier data are the same as in Fig. 7 where (a) shows signal and pump output power and (b) shows the signal gain.

less and less amplification, until the gain converges to its "dynamic" steady-state value, with an average output power on the channels necessarily below the pump $\mathrm{CW}$ power.

Fig. 8 shows the gain dynamics starting at steady state for a train of pulses of peak input power $-2 \mathrm{dBm}$, pulse duration $T_{p}=170 \mathrm{~ns}$, repetition period $T_{r}=2 T_{p}$ (one pulse every two slots), and the same amplifier data as in Fig. 7. The first pulses have a strong power sag, but as the gain decreases, subsequent pulses experience less gain, and thus the tilt on the gain decreases, to essentially vanish at dynamic equilibrium in this case of "short" pulses.

Fig. 9 shows that flat gain at equilibrium is not always present, and that the packet duration is the discriminating parameter. While for short packets, e.g., ATM cells at 2.5 $\mathrm{Gb} / \mathrm{s}$, the residual power sag at dynamic equilibrium is less than $0.5 \mathrm{~dB}$, for longer packets of duration $T_{p}=2.83 \mu \mathrm{s}$, e.g., ATM cells at $150 \mathrm{Mb} / \mathrm{s}$, the residual power sag at dynamic equilibrium can be more than $3 \mathrm{~dB}$. Note that to speedup the numerical computations and observe the solution of (5) close to dynamic equilibrium when a pulse train of peak power $Q_{s}$ is feeding the amplifier, we start with initial conditions $r(0)$ found from (6) with CW input signal $\bar{Q}_{s} \triangleq\left(T_{p} / T_{r}\right) Q_{s}$.

It is now interesting to find, for a given cell duration $T_{p}$, how the gain swing varies with the repetition frequency $f_{r} \triangleq 1 / T_{r}$. Ko et al., have experimentally measured such curves for 100 ns duration pulses ([12, Fig. 6]), but the gain swing for such short pulses is not noticeable. Solving (5) numerically for a large set of repetition frequencies is a long task. In Appendix C we resort to the exponential approximation (19) to find the gain sag across the cell as a function of the repetition rate. Using the algorithm in Appendix $\mathrm{C}$, we obtain the upper and lower values $G_{U}$ and $G_{L}$ of the dB-gain, shown in Fig. 10 versus cell repetition rate $R_{r}$, for (a) ATM cells at $2.5 \mathrm{~Gb} / \mathrm{s}$ 

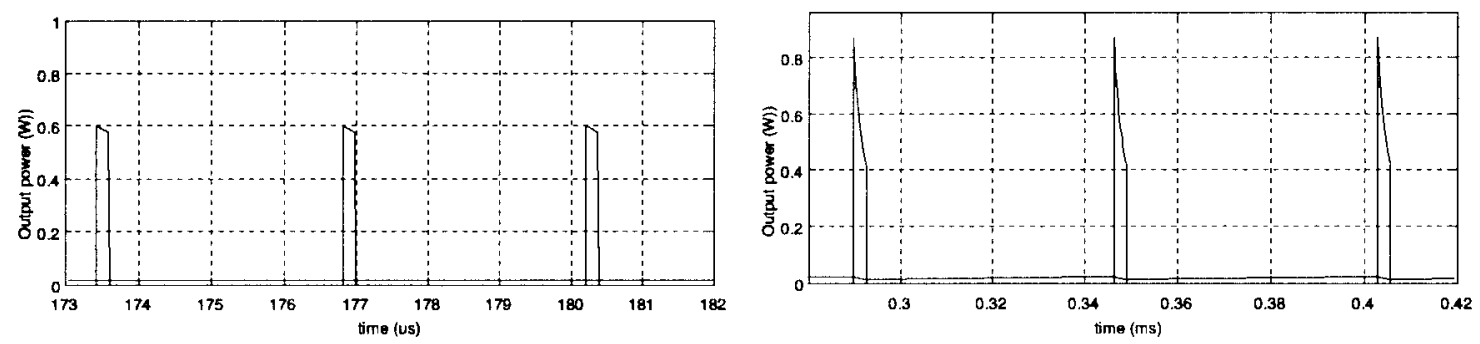

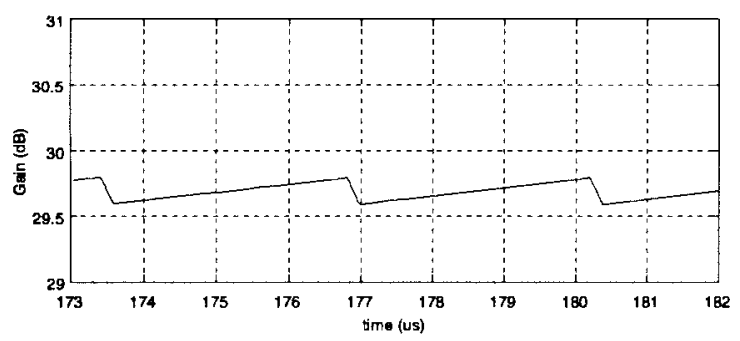

(a)

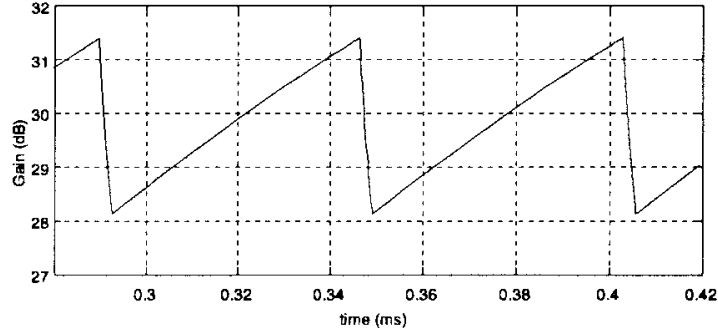

(b)

Fig. 9. Numerical solution of (5) giving the system time evolution at dynamic equilibrium. One input train of cells of peak input power $-2 \mathrm{dBm}$, one cell every 20 slots. (a) ATM cells at $2.5 \mathrm{~Gb} / \mathrm{s}$ and (b) ATM cells at $150 \mathrm{Mb} / \mathrm{s}$. Amplifier data is the same as in Fig. 7. The top graphs show signal and pump output power and the bottom graphs show the signal gain.

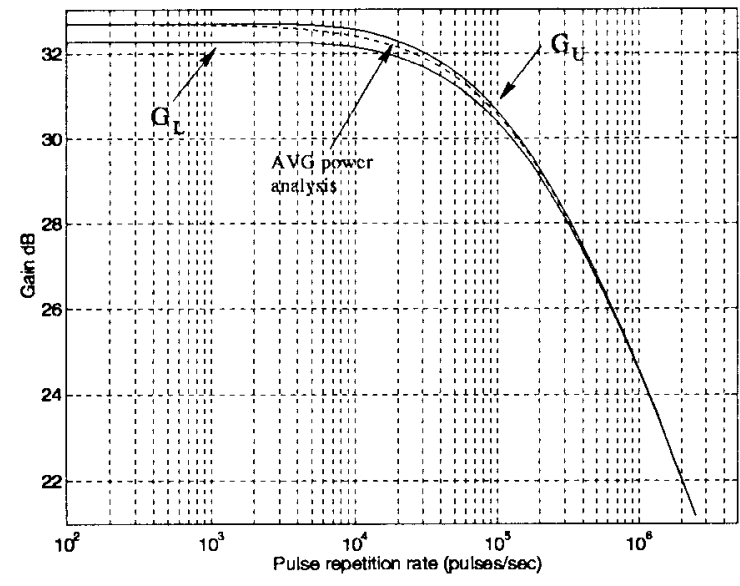

(a)

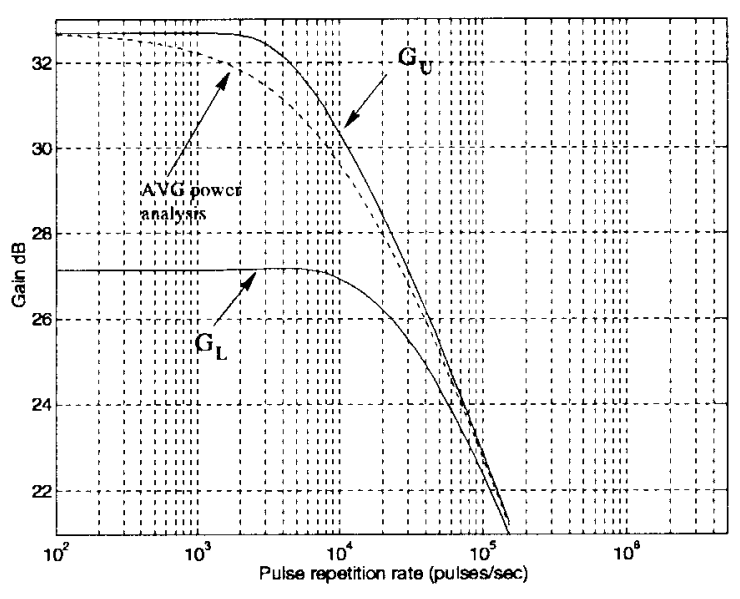

(b)

Fig. 10. Upper and lower values $G_{U}$ and $G_{L}$ of dB-gain across cells versus cell repetition rate $R_{r}$. (a) ATM cells at $2.5 \mathrm{~Gb} / \mathrm{s}$ and (b) ATM cells at $150 \mathrm{Mb} / \mathrm{s}$. Pump power $18.4 \mathrm{dBm}$, signal peak input power $-2 \mathrm{dBm}$.

and (b) ATM cells at $150 \mathrm{Mb} / \mathrm{s}$. The pump power is $18.4 \mathrm{dBm}$, and the signal peak input power is $-2 \mathrm{dBm}$.

At low repetition rates the gain sag across the cell is larger. The asymptotic value for $R_{r} \rightarrow 0$ is found easily, by considering that in such case the reservoir has time to completely recover after each pulse. Hence, $G_{U}$ tends to the small-signal gain. $G_{L}$ tends to the gain value observed in an isolated pulse, and is lower for longer pulse duration. Also shown in the figure is the average power analysis, i.e., a steadystate gain analysis using (6), assuming a CW input signal of flux $\bar{Q}_{s}$. We note that upper and lower gain values converge to the average power analysis value as the repetition frequency is increased. As already observed, for ATM cells at $150 \mathrm{Mb} / \mathrm{s}$ the gain sag can be as extreme as $6 \mathrm{~dB}$ for low repetition rates.

So far we have learned that gain dynamics can give large power swings across the cells if long cell interarrival times, or lulls, give the amplifier enough time to recover. We conclude this section by showing in Fig. 11 the effect of such lulls in an eight-channel system feeding an amplifier, with channels at wavelengths from 1538 to $1560 \mathrm{~nm}$, with $2 \mathrm{~nm}$ spacing. The amplifier has the same parameters as in Section IV. Each channel $j$ is carrying a periodic train of ATM cells of repetition time $T_{r}(j)$. The bit rate in the cells is $150 \mathrm{Mb} / \mathrm{s}$. All channels are aligned to the slot time at the input of the amplifier. We start the reservoir close to dynamic equilibrium, obtained by the average power analysis. All eight channels start at the first slot at time " 0 " with a cell.

We chose the repetition times of the trains on the channels to be $9,10,12,15,18,20,30$, and 36 slots, respectively, so that after 180 slot times (the least common multiple of the repetition times) all eight trains have again overlapping cells. This scheme artificially creates periodic lulls after each 


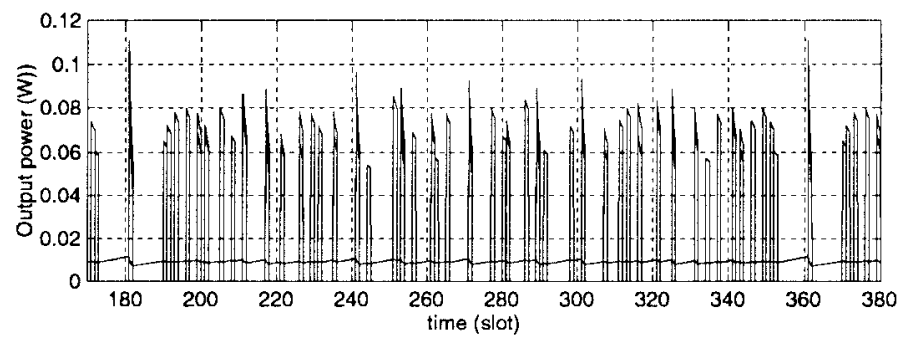

(a)

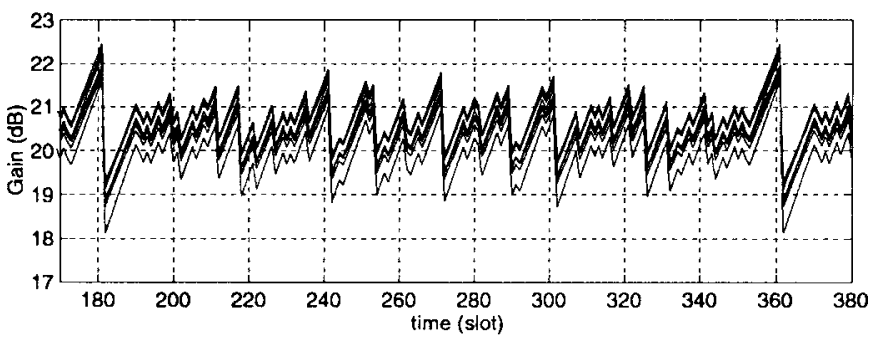

(b)

Fig. 11. Numerical solution of (5), in the case of eight pulsed channels. Pulses are ATM cells at $150 \mathrm{Mb} / \mathrm{s}$ one slot is $2.8 \mu \mathrm{s}$ and the pump is 18.4 $\mathrm{dBm}$. The peak input power is $-2 \mathrm{dBm} /$ channel. where (a) shows pump and signal output powers and (b) shows the gain at the eight signal wavelengths.

coincidence slot, every 180 slot times, of duration given by the smallest repetition time, in this case eight slots. The top graph of Fig. 11 shows pump and signal output powers. Overlapping cells at different wavelengths are visible in the figure. The bottom graph shows the gain at the eight signal wavelengths.

\section{COnClusions}

In this paper, we further simplify the ODE for the dopedfiber amplifier gain dynamics reported in [1], bringing into greater evidence the physical meaning of the amplification process, and greatly enhancing the utility of the ODE as an analysis and design tool. We find that the doped-fiber amplifier dynamics are connected to the depletion and the refill of the reservoir of excited ions in the amplifier. While the refill process is mainly contributed by the pump, and is a process in which one pump photon can at most excite one ion, the depletion process is mainly caused by the signals, and is an avalanche process connected to stimulated emission: one signal input photon can "burn" a very large number of excited ions in the reservoir. Thus, the time scales connected to the depletion process can be extremely fast, while those connected to the refill process are slow and depend on the pump power and the total number of dopant ions. In any case, the amplifier dynamics are essentially independent of the fluorescence time. An important consequence of the above is that channel addition causes much faster transients than channel dropping in WRON's.

Perhaps the best scenario to observe the amplifier gain dynamics is in multichannel packet switching applications, where signals are kept at a high power level to avoid optical SNR degradations when multiple amplifiers must be crossed before reaching destination. We have given figures of the gain swing across packets in such environment.
The present analysis is based on the assumptions of the model in [1] and [5], which neglects both excited state absorption and saturation induced by the amplified spontaneous emission (ASE) produced inside the amplifier. There are several ways to include such ASE contributions in the model [8], [9], and future work will address this issue. However, the main findings of the present work will be valid as long as the signal powers are large and the main contribution to amplifier saturation.

\section{APPENDIX A}

\section{EXPONENTIAL APPROXIMATION: DERIVATION}

In Section VII, we gave an exponential approximation to the actual solution of (5) for step-like driving signals. To give an analytical justification to the approximation, we rewrite (5) by adding and subtracting $r(0)$ in the log-gain exponent so that for $t>0$

$\dot{r}(t)=-\frac{r(t)}{\tau}+\sum_{j=0}^{N} Q_{j}^{\mathrm{in}}(t)\left\{1-e^{\left[B_{j} r(0)-A_{j}\right]} e^{B_{j}[r(t)-r(0)]}\right\}$.

Define the deviation from $r(0)$ as $r_{\Delta} \triangleq r(t)-r(0)$. Now expand the exponential term in (25) in a taylor series around $r(0)$

$$
\begin{aligned}
\dot{r}(t)= & -\frac{r(t)}{\tau}+\sum_{j=0}^{N} Q_{j}^{\text {in }}(t) \\
& \cdot\left(1-e^{\left(B_{j} r(0)-A_{j}\right)} \sum_{k=0}^{\infty} \frac{B_{j} r_{\Delta}^{k}}{k !}\right) .
\end{aligned}
$$

We can then write

$$
\begin{aligned}
\dot{r}_{\Delta}(t)= & \left\{-\frac{r(0)}{\tau}+\sum_{j=0}^{N} Q_{j}^{\mathrm{in}}(k)\left[1-e^{G_{j}(0)}\right]\right\} \\
& -\left[\frac{1}{\tau}+\sum_{j=0}^{N} Q_{j}^{\mathrm{in}}(t) e^{G_{j}(0)} B_{j}\right] r \Delta(t)+\mathcal{O}(r \Delta) \\
= & \dot{r}\left(0^{+}\right)-\left[\frac{1}{\tau}+\sum_{j=0}^{N} Q_{j}^{\mathrm{in}}(t) e^{G_{j}(0)} B_{j}\right] r \Delta(t)+\mathcal{O}(r \Delta)
\end{aligned}
$$

where $G_{j}(0)=B_{j} r(0)-A_{j}$, and $\mathcal{O}(r \Delta)$ represents terms vanishing faster than $r \Delta$ as $r \Delta \rightarrow 0$. If we neglect the $\mathcal{O}(r \Delta)$ terms, and the drive signals $Q_{j}^{\text {in }}(t)$ are time-independent for $t>0$, then (27) becomes a linear ODE whose solution is

$$
r_{e}(t)=r(0)+\tau_{e} \dot{r}\left(0^{+}\right)\left(1-e^{-t / \tau_{e}}\right)
$$

with time constant

$$
\tau_{e}=\left[\frac{1}{\tau}+\sum_{j=0}^{N} Q_{j}^{\text {in }} e^{G_{j}(0)} B_{j}\right]^{-1} .
$$

This time constant is also given in [4, eq. (28)].

Recalling our equivalent circuit model, note from (29) that we can interpret $\sum_{j=0}^{N} Q_{j}^{\text {in }} e^{G_{j}(0)} B_{j}$ as an equivalent 
conductance $g_{e q}$ which is in parallel with $1 / \tau$ to give the effective circuit conductance $1 / \tau_{e}$ seen by the capacitor, the one determining the circuit dynamics. Once again, it is clear that the system dynamics very weakly depend on the "resistor" $\tau$, since this is in parallel to the usually much smaller resistor $1 / g_{\text {eq }}$.

Note that both approximation (19) and (28) give the correct values for the derivative at time zero despite differing time constants. However, only (19) gives the correct asymptotic value of the reservoir in steady state. The exponential form (28) is a good approximation for small values of $t$, while the approximation in Section VII extends to asymptotic values as well.

\section{APPENDIX B}

\section{Gain Peaking In AMPLIFIER Chains}

The gain spread in each amplifier along a chain of amplifiers leads to gain peaking [10], i.e., to the fact that one channel tends asymptotically to a fixed output power at the output of each amplifier down the chain, while all other powers decrease and eventually disappear. Gain peaking is the dominant feature characterizing chains of fiber amplifiers, the one that has most impact on system performance.

To derive gain peaking analytically, consider a chain as described in Section VIII. Recall that in this analysis, we neglect ASE. Consider the next steady state after the power discontinuity. The reservoir of the $m$ th amplifier satisfies (6)

$$
r_{m}^{s s}=\tau \sum_{j=0}^{N} Q_{m j}^{\text {in }}\left(1-e^{B_{j} r_{m}^{s s}-A_{j}}\right)
$$

$Q_{m j}^{\mathrm{in}}(t \rightarrow \infty)$ is obtained from (21), giving (31) shown at the bottom of the page.

Now we look for the limit value of $r_{m}^{s s}$ as $m \rightarrow \infty$. Suppose such a value exists, and denote it as $r_{\infty}^{s s} \triangleq \lim _{m \rightarrow \infty} r_{m}^{s s}$. It is easy to show that under this assumption $\lim _{m \rightarrow \infty}(1 / m) \sum_{i=1}^{m} r_{i}^{s s}=r_{\infty}^{s s}$. Now take $\lim _{m \rightarrow \infty}$ of both sides of (31). Consider the limit of the exponent on the right-hand side (RHS)

$$
\lim _{m \rightarrow \infty}(m-1)\left[B_{j} \frac{\sum_{i=1}^{m-1} r_{i}^{s s}}{m-1}-\left(A_{j}+\ln L_{I}\right)\right] .
$$

In order to get a finite value of the exponent for channel $j$, we need

$$
\lim _{m \rightarrow \infty} \frac{1}{m-1} \sum_{i=1}^{m-1} r_{i}^{s s}=r_{\infty}^{s s}=\frac{A_{j}+\ln L_{I}}{B_{j}} .
$$

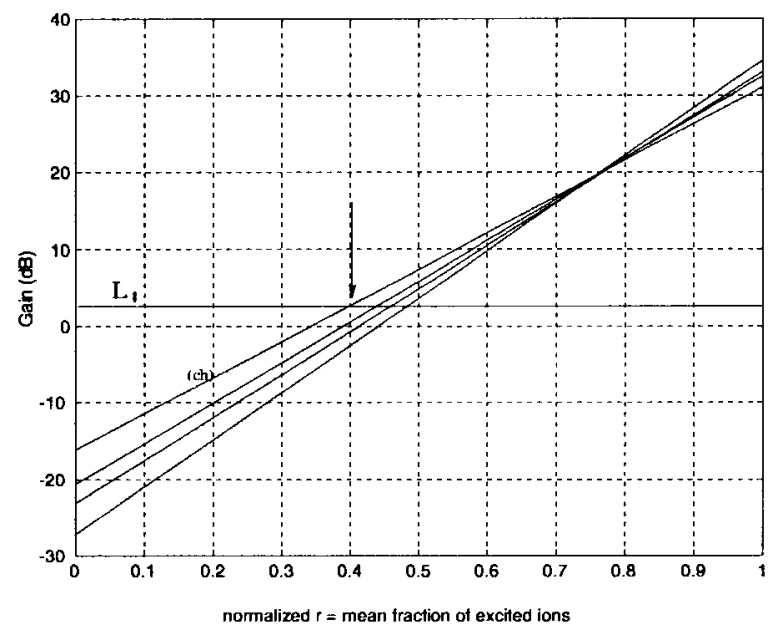

Fig. 12. Graphical determination of steady state (normalized) reservoir value $r_{\infty}^{s s}$ and surviving channel $c h$ along an infinite chain of interamplifier loss $L_{I}$.

If instead we have $r_{\infty}^{s s}<\left(A_{j}+\ln L_{I}\right) / B_{j}$, then the limit of the exponent is $-\infty$.

Thus, for interamplifier loss $L_{I}$, the limit value $r_{\infty}^{s s}$ is given by, as shown in Fig. 12, the largest value of $r$ such that the log-gain of a specific channel $c h$ equals the interamplifier loss $\ln L_{I}$, and the log-gain of all other channels $j$ is less than $\ln L_{I}$. Only channel $c h$ survives in the infinite chain, its gain being equal to the loss, while all other channels vanish, having gain lower than the loss. The limit value of the reservoir is found to be

$$
r_{\infty}^{s s}=\min _{\{j=1, \cdots, N\}}\left\{\frac{A_{j}+\ln L_{I}}{B_{j}}\right\}
$$

and the surviving channel $c h$ is the one that achieves the minimum.

\section{APPENDIX C}

This Appendix derives the approximate dynamic equilibrium extremum values of the reservoir when the amplifier is pumped by a CW pump with flux $Q_{p}$ and is fed by a signal pulse train of pulse duration $T_{p}$, repetition time $T_{r}$, and peak input flux $Q_{s}$.

At dynamic equilibrium, the upper and lower values of the reservoir $r_{U} \triangleq r\left(t_{U}\right)$ and $r_{L} \triangleq r\left(t_{L}\right)$ are related to the pulse duration $T_{p}$ and the idle time between pulses $T_{i}=T_{r}-T_{p}$ as shown in Fig. 13. To find $r_{U}$ and $r_{L}$ at equilibrium we use the following numeric algorithm.

Start with an initial guess $r_{U}=r_{\text {avg }}^{s s}(\infty)$, where $r_{\text {avg }}^{s s}(\infty)$ is the steady state value found from (6) with input fluxes $Q_{p}$ and $Q_{s}$.

$$
\frac{r_{m}^{s s}}{\tau}=Q_{p}\left(1-e^{B_{p} r_{m}^{s s}-A_{p}}\right)+\sum_{j=1}^{N} Q_{j}^{\text {in }}\left(0^{+}\right) \exp \left((m-1)\left\{B_{j}\left[\sum_{i=1}^{m-1} r_{i}^{s s} /(m-1)\right]-\left(A_{j}+\ln L_{I}\right)\right\}\right)\left(1-e^{B_{j} r_{m}^{s s}-A_{j}}\right)
$$




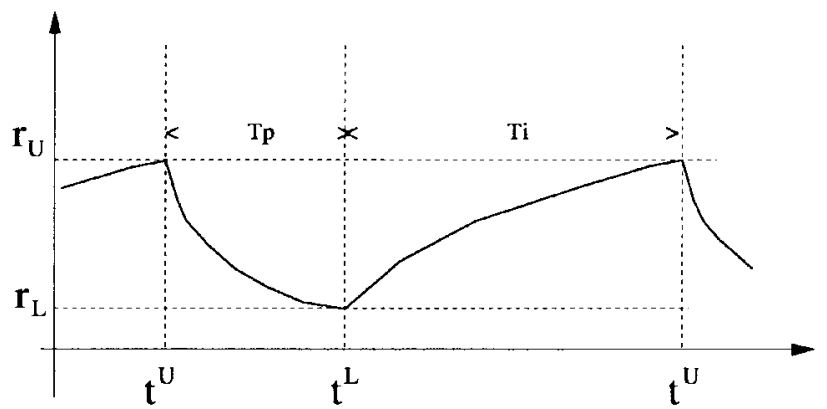

Fig. 13. Dynamic equilibrium.

1) From (15), we get

$$
\dot{r}\left(t_{U}^{-}\right)=-\frac{r\left(t_{U}\right)}{\tau}+Q_{p}\left(1-e^{B_{p} r_{U}-A_{p}}\right)
$$

since $Q_{s}\left(t_{U}^{-}\right)=0$.

2) From (16), we get

$$
\dot{r}\left(t_{U}^{+}\right)=\dot{r}\left(t_{U}^{-}\right)+Q_{s}\left(1-e^{B_{s} r_{U}-A_{s}}\right)
$$

since $Q_{s}$ is the only channel with step variation $Q_{s}$ at $t=t_{U}^{+}$.

$3)$ In the exponential approximation of the gain dynamics, from (20) we get

$$
\tau_{e}(U)=\frac{\left[r_{U}^{s s}(\infty)-r_{U}\right]}{\dot{r}\left(t_{U}^{+}\right)}
$$

where $r_{U}^{s s}(\infty)$ is the steady state value found from (6) with input fluxes $Q_{p}$ and $Q_{s}$. Then we set

$$
r_{L}=r_{U}^{s s}(\infty)+\left[r_{U}^{s s}(\infty)-r_{U}\right] e^{-T_{p} / \tau_{e}(U)} .
$$

4) Update the reservoir derivative at $t_{L}$ using (15)

$$
\begin{aligned}
\dot{r}\left(t_{L}^{-}\right)= & -\frac{r\left(t_{L}\right)}{\tau}+Q_{p}\left(1-e^{B_{p} r_{L}-A_{p}}\right) \\
& +Q_{s}\left(1-e^{B_{p} r_{L}-A_{p}}\right)
\end{aligned}
$$

since both $Q_{s}$ and $Q_{p}$ are present at $t_{L}^{-}$.

5) From (16), we get

$$
\dot{r}\left(t_{L}^{+}\right)=\dot{r}\left(t_{L}^{-}\right)-Q_{s}\left(1-e^{B_{s} r_{L}-A_{s}}\right)
$$

since $Q_{s}$ is the only channel with step variation $-Q_{s}$ at $t=t_{L}^{+}$.

6) In the exponential approximation of the gain dynamics, from (20) we get

$$
\tau_{e}(L)=\frac{r_{L}^{s s}(\infty)-r_{L}}{\dot{r}\left(t_{L}^{+}\right)}
$$

where $r_{L}^{s s}(\infty)$ is the steady state value found from (6) with input flux $Q_{p}$ only (pulse absent). Then we set

$$
r_{U}=r_{L}^{s s}(\infty)+\left[r_{L}^{s s}(\infty)-r_{L}\right] e^{-T_{I} / \tau_{e}(L)} .
$$

7) Repeat 1)-6) until $r_{U}$ and $r_{L}$ converge.
The same algorithm can be applied using the linear approximation in (17) instead. The values obtained by the linear approximation tend to converge to those obtained by the exponential approximation for high repetition rates, although the exponential approximation always gives values closer to the actual ones.

\section{REFERENCES}

[1] Y. Sun, G. Luo, J. L. Zyskind, A. A. M. Saleh, A. K. Srivastava, and J. W. Sulhoff, "Model for gain dynamics in erbium-doped fiber amplifiers," Electron. Lett., vol. 32, pp. 1490-1491, Aug. 1996.

[2] Y. Sun, A. K. Srivastava, J. L. Zyskind, J. W. Sulhoff, J. Wolf, and R. W. Tkach, "Fast power transients in WDM optical networks with cascaded EDFA's," Electron. Lett., vol. 33, pp. 313-314, Feb. 1997.

[3] A. K. Srivastava, J. L. Zyskind, and J. W. Sulhoff, "EDFA transient response to channel loss in WDM transmission system," IEEE Photon. Technol. Lett., vol. 9, pp. 386-388, Mar. 1997.

[4] Y. Sun, A. A. M. Saleh, J. L. Zyskind, D. L. Wilson, A. K. Srivastava, and J. W. Sulhoff, "Time dependent perturbation theory and tones in cascaded erbium-doped fiber amplifier systems," J. Lightwave Technol., vol. 15, pp. 1083-1087, July 1997.

[5] A. A. M. Saleh, R. M. Jopson, J. D. Evankov, and J. Aspell, "Modeling of gain in erbium-doped fiber amplifiers," IEEE Photon. Technol. Lett., vol. 2, pp. 714-717, Oct. 1990.

[6] I. M. I. Habbab, A. A. M. Saleh, and P. K. Runge, "Erbium-doped fiber amplifiers: Linear approximations," J. Lightwave Technol., vol. 13, pp. 33-36, Jan. 1995.

[7] Y. Sun, J. L. Zyskind, and A. K. Srivastava, "Average inversion level, modeling, and physics of erbium-doped fiber amplifiers," J. Select. Areas Quantum Electron., Special Issue on Fiber Amplifiers and Lasers, to be published.

[8] T. Georges and E. Delevaque, "Analytic modeling of high-gain erbiumdoped fiber amplifiers," Opt. Lett., vol. 17, pp. 1113-1115, Aug. 1992.

[9] R. M. Jopson and A. A. M. Saleh, "Modeling of gain and noise in erbium-doped fiber amplifiers," in Proc. SPIE Fiber Laser Sources Amplifiers III, 1991, vol. 1581, pp. 114-119.

[10] E. Desurvire, Erbium-Doped Fiber Amplifiers. New York: Wiley, 1994.

[11] T. Otani, K. Goto, T. Kawazawa, H. Abe, and M. Tanaka, "Effect of span loss increase on the optically amplified communication system," $J$. Lightwave Technol., vol. 15, pp. 737-742, May 1997.

[12] K. Y. Ko, M. S. Demokan, and H. Y. Tam, "Transient analysis of erbium-doped fiber amplifiers," IEEE Photon. Technol. Lett., vol. 6, pp. 1436-1438, Dec. 1994.

[13] G. W. Agrawal, Nonlinear Fiber Optics, 2nd ed. New York: Academic, 1995.

Alberto Bononi (M'90), for a biography, see p. 500 of the April 1998 issue of this JOURNAL.

Leslie A. Rusch (S'91-M'94) was born in Chicago, IL. She received the B.S.E.E. degree from the California Institute of Technology, Pasadena, in 1980 and the M.A. and Ph.D. degrees in electrical engineering from Princeton University, Princeton, NJ, in 1992 and 1994, respectively.

She is currently an Associate Professor of Electrical Engineering at l'Université Laval, P.Q., Canada. She occupies a chair in optical communications jointly sponsored by the Natural Science and Engineering Research Council of Canada and Québec-Téléphone. Her research interests include fiber optic communications, transient gain analysis of erbium-doped fiber amplifiers, wireless communications, spread-spectrum communications, and code division multiple access for radio and optical frequencies. 